\title{
Identification of novel variants in the $L D L R$ gene in Russian patients with familial hypercholesterolemia using targeted sequencing
}

\author{
VALENTINA V. MIROSHNIKOVA ${ }^{1}$, OLGA V. ROMANOVA ${ }^{2,3}$, OLGA N. IVANOVA ${ }^{4}$, MIKHAIL A. FEDYAKOV ${ }^{2}$, \\ ALEXANDRA A. PANTELEEVA ${ }^{1,5-7}$, YURY A. BARBITOFF ${ }^{3,7}$, MARIA V. MUZALEVSKAYA ${ }^{8,9}$, \\ SOREJYA A. URAZGILDEEVA ${ }^{8,9}$, VICTOR S. GUREVICH ${ }^{8,9}$, STANISLAV P. URAZOV $^{2}$, SERGEY G. SCHERBAK ${ }^{2}$, \\ ANDREY M. SARANA ${ }^{9}$, NATALIA A. SEMENOVA ${ }^{4}$, INGA V. ANISIMOVA ${ }^{4}$, DARYA M. GUSEVA ${ }^{4}$, \\ SOFYA N. PCHELINA ${ }^{1,5,6}$, ANDREY S. GLOTOV ${ }^{2,3}$, EKATERINA Y. ZAKHAROVA ${ }^{4}$ and OLEG S. GLOTOV GL $^{2,3}$ \\ ${ }^{1}$ Laboratory of Human Molecular Genetics, Molecular and Radiation Biophysics Department, \\ Petersburg Nuclear Physics Institute, National Research Center 'Kurchatov Institute', Gatchina 188300; \\ ${ }^{2}$ Genetic Laboratory of City Hospital No. 40, Saint-Petersburg, 197706; ${ }^{3}$ Department of Genomic Medicine, \\ D.O. Ott Research Institute of Obstetrics, Gynaecology and Reproduction, Saint-Petersburg 199034; \\ ${ }^{4}$ Laboratory of Hereditary Metabolic Diseases and Counselling Unit of Federal State Budgetary Institution \\ 'Research Centre for Medical Genetics', Moscow 115522; ${ }^{5}$ Kurchatov Complex of NBICS Nature-Like Technologies of \\ National Research Center 'Kurchatov Institute', Moscow 123182; ${ }^{6}$ Molecular-Genetic and Nanobiological Technology \\ Department of Scientific Research Center, Pavlov First Saint-Petersburg State Medical University, \\ Saint-Petersburg 197022; ${ }^{7}$ Bioinformatics Institute, Saint-Petersburg 197342; ${ }^{8}$ Department for Atherosclerosis and \\ Lipid Disorders of North-Western District Scientific and Clinical Center Named After L.G. Sokolov FMBA, \\ Saint-Petersburg 194291; ${ }^{9}$ Medical Faculty of Saint-Petersburg State University, Saint-Petersburg 199034, Russian Federation
}

Received July 3, 2020; Accepted October 9, 2020

DOI: 10.3892/br.2020.1391

\begin{abstract}
Familial hypercholesterolemia (FH) is caused by mutations in various genes, including the $L D L R, A P O B$ and PSCK9 genes; however, the spectrum of these mutations in Russian individuals has not been fully investigated. In the present study, mutation screening was performed on the $L D L R$ gene and other $\mathrm{FH}$-associated genes in patients with definite or possible FH, using next-generation sequencing. In total, 59 unrelated patients were recruited and sorted into two separate groups depending on their age: Adult $(n=31$; median age, 49; age range, 23-70) and children/adolescent $(n=28$; median age, 11; age range, 2-21). FH-associated variants were identified in 18 adults and 25 children, demonstrating mutation detection rates of 58 and $89 \%$ for the adult and children/adolescent
\end{abstract}

Correspondence to: Dr Valentina V. Miroshnikova, Laboratory of Human Molecular Genetics, Molecular and Radiation Biophysics Department, Petersburg Nuclear Physics Institute, National Research Center 'Kurchatov Institute', 1, mkr. Orlova Roscha, Gatchina 188300, Russian Federation

E-mail: miroshnikova_vv@pnpi.nrcki.ru

Key words: next generation sequencing, familial hypercholesterolemia, $L D L R$ groups, respectively. In the adult group, 13 patients had FH-associated mutations in the $L D L R$ gene, including two novel variants [NM_000527.4: c.433_434dupG p.(Val145Glyfs*35) and c.1186G $>$ C p.(Gly396Arg)], 3 patients had $A P O B$ mutations and two had $A B C G 5 / G 8$ mutations. In the children/adolescent group, 21 patients had FH-causing mutations in the $L D L R$ gene, including five novel variants [NM_000527.4: c.325T $>$ G p.(Cys109Gly), c.401G $>$ C p.(Cys134Ser), c.616A >C p.(Ser206Arg), c.1684_1691delTGGCCCAA p.(Pro563Hisfs*14) and c.940+1_c.940+4delGTGA], and 2 patients had $A P O B$ mutations, as well as $A B C G 8$ and $L I P A$ mutations, being found in different patients. The present study reported seven novel $L D L R$ variants considered to be pathogenic or likely pathogenic. Among them, four missense variants were located in the coding regions, which corresponded to functional protein domains, and two frameshifts were identified that produced truncated proteins. These variants were observed only once in different patients, whereas a splicing variant in intron 6 (c.940+1_c.940+4delGTGA) was detected in four unrelated individuals. Previously reported variants in the $L D L R$, $A P O B, A B C G 5 / 8$ and LIPA genes were observed in 33 patients. The $L D L R$ p.(Gly592Glu) variant was detected in 6 patients, representing $10 \%$ of the $\mathrm{FH}$ cases reported in the present study, thus it may be a major variant present in the Russian population. In conclusion, the present study identified seven novel variants of the $L D L R$ gene and broadens the spectrum of mutations in FH-related genes in the Russian Federation. 


\section{Introduction}

Familial hypercholesterolemia (FH) inheritable disorder of abnormal low-density lipoprotein (LDL) metabolism that is characterized by elevated plasma concentrations of total cholesterol (TC) and LDL cholesterol (LDL-C), xanthomas (cholesterol deposits in the skin and tendons) and an increased risk of premature coronary artery disease (CAD). Homozygous $\mathrm{FH}$ affects 1 in 1,000,000 individuals, and the frequency of heterozygous FH varies from 1 in 200 to 1 in 500 individuals, depending on the population (1).

Monogenic FH is caused by defects in several genes that encode proteins involved in LDL uptake and catabolism (LDLR, APOB, PCSK9 and LDLRAPI) (1). The majority of patients with autosomal dominant $\mathrm{FH}$ harbor mutations in the LDL receptor gene. To date, $>2,000 \mathrm{FH}$-causing variants have been reported in LDLR (2). APOB and PCSK9 mutations account for a smaller percentage of autosomal dominant $\mathrm{FH}$ cases (3). Recessive forms of $\mathrm{FH}$ are associated with variants in $L D L R A P 1$, which encodes for the LDLR adapter protein 1 (1).

Hypercholesterolemia may be associated with other rare disorders of lipid metabolism, which have very similar clinical presentations; one example is sitosterolemia, which is caused by mutations in genes encoding either of two ATP-binding cassette (ABC) transporters, ABCG5 or ABCG8, which limits intestinal absorption and promotes biliary excretion of sterols (4). Next-generation sequencing (NGS) studies have shown that rare mutations in the $A B C G 5, A B C G 8, A P O E$ and LIPA genes can cause a FH-like phenotype (5).

Despite being one of the most common genetic disorders, FH still remains largely undetected and untreated worldwide $(2,5)$. Screening during childhood may enhance the potential identification of individuals with the condition before establishment of cardiovascular pathologies (6). As lipoprotein metabolism in children is influenced by fewer environmental factors than it is for adults, the difference in LDL-C levels between children with and without $\mathrm{FH}$ is more pronounced (7). Pediatric FH is diagnosed phenotypically by the presence of elevated LDL-C levels, in addition to a family history of premature CAD, high baseline TC levels in one parent and/or a FH-causing mutation (6). It should be noted that there are no universal criteria for LDL-C cut-offs in the case of pediatric diagnosis of $\mathrm{FH}$. Although the widely used Simon Broome criteria proposes an LDL-C cut-off of $4 \mathrm{mmol} / \mathrm{l}(155 \mathrm{mg} / \mathrm{dl})$ for individuals under $16(8)$, another suggestion is $3.5 \mathrm{mmol} / 1$ $(140 \mathrm{mg} / \mathrm{dl})(9,10)$. The younger the child with suspected FH, the lower LDL-C should be expected to be (11). LDL-C levels can fluctuate greatly during childhood, and in the case of LDL-C levels between 2.7-3.5 mmol/1 (100-140 mg/dl), it is highly recommended that the patient is followed-up for at least a few years by the Japan Pediatric Society and Japan Atherosclerosis Society (9). As FH can only be diagnosed definitively in the presence of xanthomas, which are rarely observed in children and adolescents (7), genetic screening is a fundamental diagnostic tool. The heterogeneity of FH-causing variants and the total length of coding regions of FH-associated genes supports the use of NGS-sequence-based mutation screening as a primary methodology for diagnostics and improving the overall mutation detection rate.
The spectrum and prevalence of FH-related mutations remains to be studied in Russia. Previous studies have used limited genetic screening methods based on PCR and Sanger sequencing to study specific relevant genes, such as $L D L R$ and $A P O B$ (12-14). The aim of the present study was to adapt an NGS-based method for molecular FH diagnosis in Russian individuals and compare its efficiency in different age groups. In the present study, mutation screening in two groups of patients with suspected FH was presented: The adult group and the children/adolescent group. NGS was also used to increase the mutation detection rate.

\section{Patients and methods}

Subjects. The present study was approved by the Ethics Committees of Center for Atherosclerosis and Lipid Disorders of North-Western District Scientific and Clinical Center Named After L.G. Sokolov, Medical Faculty of Saint-Petersburg State University, City Hospital No. 40 (St. Petersburg, Russia) and Research Centre for Medical Genetics (Moscow, Russia), where patients were treated, and genetic analysis was performed. Written informed consent was obtained from all patients or the children's legal representatives prior to the beginning of the study.

A total of 59 unrelated citizens from Saint-Petersburg and Moscow (29 male/30 female) with suspected FH were enrolled in the present study. Clinical data were collected, including the prior lipid levels, family and personal history of dyslipidemia and the presence of premature atherosclerotic cardiovascular disease (ASCVD), as well as the presence of tendon/skin xanthomas and lipoic corneal arcus. Demographic characteristics and clinical features of the groups are presented in Table I. The recorded TC and LDL-C values, independent of pre- or post-treatment, were used in the present study. Depending on the age, patients were sorted into two separate groups: The adult group ( $\geq 21$ years old) and the children/adolescent group $(<21)(15)$. As children and adolescents were included, the Simon Broome criteria was used. Exclusion criteria were the presence of thyroid dysfunction, nephrotic syndrome, autoimmune disease or primary biliary cirrhosis.

The adult group included 31 patients (12 males and 19 females; median age, 49; age range, 23-70) who fulfilled the Simon Broome criteria for definite/possible FH (8). The children/adolescent group included 28 children and adolescents (17 males and 11 females; median age, 11; age range, 2-21) who mostly met the strict criteria regarding lipid levels (TC $>7.5 \mathrm{mmol} / 1$ or LDL-C $>4.9 \mathrm{mmol} / 1$ if $>16$ years; TC $>6.7 \mathrm{mmol} / 1$ or LDL-C $>4.0 \mathrm{mmol} / 1$ if $<16$ years). Children with LDL-C $<4.0 \mathrm{mmol} / 1$ were also included in the study as the diagnosis of $\mathrm{FH}$ in children can be based on age and sex adjusted LDL-C levels, with the 95th percentile being $3.5 \mathrm{mmol} / 1$ for boys and $3.8 \mathrm{mmol} / \mathrm{l}$ for girls (16). A previous clinical study stated that the LDL-C cut-off level may be even lower $(3.4 \mathrm{mmol} / \mathrm{l})$ if a first-degree relative shows increased TC and LDL-C levels, or has been diagnosed with ASCVD (17). A total of $86 \%$ individuals from the children/adolescent group represented families with a history of hypercholesterolemia and/or ASCVD, but never applied for genetic testing. For several patients included in the study, elevated TC levels 
Table I. Clinicopathological and demographic characteristics of the recruited cohort.

\begin{tabular}{|c|c|c|}
\hline Characteristic & Adult, $\mathrm{n}=31$ & Children/adolescent, $n=28$ \\
\hline Age, years ${ }^{\mathrm{a}}$ & $47.4 \pm 14.1$ & $11.0 \pm 5.1$ \\
\hline Age range, years & $23-70$ & $2-21$ \\
\hline Male, n (\%) & $12(39)$ & $17(61)$ \\
\hline Female, n (\%) & $19(61)$ & $11(39)$ \\
\hline Family history, n (\%) & $23(74)$ & $24(86)$ \\
\hline Maximal total cholesterol, $\mathrm{mmol} / \mathrm{l}^{\mathrm{a}}$ & $11.0 \pm 2.2$ & $9.3 \pm 1.4$ \\
\hline LDL cholesterol, mmol// ${ }^{\mathrm{a}}$ & $6.8 \pm 2.5$ & $6.7 \pm 1.8$ \\
\hline Tendon xanthomas, n (\%) & $22(71)$ & $0(0)$ \\
\hline Lipoic corneal arcus, $\mathrm{n}(\%)$ & $2(6)$ & $0(0)$ \\
\hline Clinical and instrumental manifestations of ASCVD, n (\%) & $17(55)$ & $0(0)$ \\
\hline Increased intima-media thickness without clinical symptoms, n (\%) & $3(10)$ & $0(0)$ \\
\hline Patients on lipid-lowering therapy, n (\%) & $31(100)$ & $1(4)$ \\
\hline
\end{tabular}

${ }^{\text {a Mean }} \pm$ standard deviation. ASCVD, atherosclerotic cardiovascular disease; LDL, low density lipoprotein.

were a consequential finding during routine biochemical examinations due to frequent respiratory infections.

$N G S$. NGS was performed as a collaboration between two genetic laboratories from Saint-Petersburg and Moscow, and the Illumina MiSeq (Illumina, Inc.) and Ion S5 (Thermo Fisher Scientific, Inc.) sequencing systems were used, respectively. Genomic DNA (gDNA) was extracted from whole blood using the Magna Pure system (Roche Diagnostics) or with the use of a Diatom DNA Prep reagent kit (Biocom) according to the manufacturer's protocols. Concentration of gDNA as well as DNA concentration of the libraries afterwards was determined using a Quantus Fluorometer ${ }^{\mathrm{TM}}$ (Promega Corporation) or Qubit $^{\mathrm{TM}}$ Fluorometer (Thermo Fisher Scientific, Inc.). gDNA was subjected to electrophoresis in $1 \%$ agarose gel and the optical density ratio was used to confirm its integrity and purity.

DNA samples were prepared for the targeted NGS covering all of the coding exons of the LDLR (NM_000527), APOB (NM_000384), PCSK9 (NM_174936), LDLRAPI (NM_015627), ABCG5 (NM_022436) and $A B C G 8$ (NM_022437) genes. The panel used for mutation screening in the children/adolescent group additionally included LIPA (NM_001127605), as LIPA disorders manifest with FH-like clinical features at a young age.

For sequencing on the Illumina platform, DNA libraries were prepared from $200 \mathrm{ng}$ using a KAPA LTP Library Preparation kit with a custom designed SeqCap ${ }^{\circledR}$ EZ Choice Library Enrichment kit [Roche Diagnostics; cat. no. KK8232 (07961880001) and 170911_HG19_gb40_cardio_EZ_HX3, respectively]. Validation of the libraries was performed on the Agilent 4200 Tape Station (Agilent Technologies, Inc.). Concentration in nmol was calculated based on the size of the libraries and concentration in $\mathrm{ng} / \mu \mathrm{l}$. Libraries were normalized to $4 \mathrm{nmol}$ before pooling and denaturation to get a final loading concentration of 12.5 pmol. Paired-end sequencing of the $150 \mathrm{bp}$ libraries was performed on an Illumina MiSeq Sequencer (Illumina, Inc.) using a MiSeq Reagent kit v2 (300 cycles) (Illumina, Inc., cat. MS-102-2002) to obtain the FastQ data.
For sequencing on the Ion S5 system, DNA libraries were constructed with the Ion AmpliSeq ${ }^{\mathrm{TM}}$ custom panel and Ion AmpliSeq $^{\mathrm{TM}}$ Library kit 2.0 (Thermo Fisher Scientific, Inc.; cat. nos. 04779971_Dyslipidemia_IAD175748_182 and 4480442 , respectively). DNA quality was confirmed by the final stage of library preparation using test PCR performed with the included manufacturer's primers to adaptors sequences. The thermocycling conditions used were as follows: $95^{\circ} \mathrm{C}$ for $40 \mathrm{sec}, 68^{\circ} \mathrm{C}$ for $35 \mathrm{sec}$ and $72^{\circ} \mathrm{C}$ for $75 \mathrm{sec}$; the number of cycles used was dependent on the library concentration. PCR results were visualized on silver-stained $8 \%$ acrylamide gel (staining time $10 \mathrm{~min}$ at $4^{\circ} \mathrm{C}$ ). Massive parallel sequencing of pooled libraries with loading concentration of $75 \mathrm{pmol}$ was performed using an Ion $540^{\mathrm{TM}}$ Chip kit (Thermo Fisher Scientific, Inc., cat. A27766) and Ion 540 ${ }^{\mathrm{TM}}$ Kit-Chef (Thermo Fisher Scientific, Inc., cat. mo. A30011) with an average amplicon length of 175 bps.

Bioinformatics analysis. The 1000 Genomes human reference genome assembly (b37) was used for data analysis (18). All samples were analyzed using a bioinformatics pipeline based on the BWA-MEM version 0.7.15-r1140, PicardTools version 2.2.2 (broadinstitute.github.io/picard/) and Genome Analysis Tool kit (GATK) version 3.5 (github. com/broadinstitute/gatk/releases) software according to the GATK Best Practices workflow (software.broadinstitute. org/gatk/best-practices/) (19,20). Target enrichment metrics were collected using the Picard CalculateHsMetrics tool. All samples included in the dataset were jointly genotyped using the GATK GenotypeGVCFs tool. Variants were hard filtered using threshold values recommended by GATK. Variant annotation was performed using SnpEff and SnpSift packages. The following resources and databases were used for variant annotation: dbSNP build 146, 1000 Genomes phase 3 (21); Exome Aggregation Consortium r. 0.3.1 (22); ClinVar version 2018-04-01 and dbNSFP version 2.9 (23). To determine splicing alterations, the NetGene2 Server (cbs. dtu.dk/services/NetGene2) was used. The frequency of the 
identified variants was additionally assessed following the Northwest Russia variant compendium (24). Novel variants were defined based on the following criteria: i) No reference SNP ID number; and ii) they had not been recorded in the public database including the Human Gene Mutations Database (HGMD; hgmd.cf.ac.uk), ClinVar or a publicly funded database for $L D L R$ mutations (LOVD; databases. lovd.nl/shared/variants/). The prediction of the pathogenicity of previously undescribed mutations was performed using the SIFT (sift.jcvi.org/), PolyPhen-2 (genetics.bwh.harvard. edu/pph2) and MutationTaster (mutationtaster.org) tools, as well as Human Splicing Finder for intronic variants (umd. be/HSF3/HSF.shtml) (25). The nomenclature of molecular variants follows the Human Genome Variation Society guidelines (varnomen.hgvs.org/). Assessment of the pathogenicity of novel sequence variants was performed taking into account the recommendations of the American College of Medical Genetics and Genomics (26). These previously developed protocols have allowed effective identification of pathogenic variants for various other hereditary diseases (27).

Variant validation. Variant validation was performed by PCR-direct sequencing. Specific primers were designed for verification in each case (Table SI). DNA sequencing was performed using the ABI BigDye Terminator 3.1 kit (Thermo Fisher Scientific, Inc.; cat. no. 4337456) on an ABI 3130xl Genetic analyzer (Saint-Petersburg) or ABI 3500 automatic sequencer (Moscow; both supplied by Applied Biosystems; Thermo Fisher Scientific, Inc.) according to the manufacturer's specifications.

\section{Results}

NGS-based genetic findings. The NGS-based technique allowed for the identification of 32 different pathogenic/likely pathogenic variants, 7 of which had not been previously reported, in 43 patients (Table II). The overall mutation detection rate was $73 \%$. Phenotypic characteristics of mutation-positive patients are reported in Tables SII and SIII. All novel variants in the $L D L R$ gene identified in this study and their pathogenicity analyses are presented in Table II, with their Sanger sequencing results shown in Fig. 1.

In the adult group, pathogenic/likely pathogenic variants were detected in $18(58 \%)$ patients: 13 Patients had FH-causing mutations in the $L D L R$ gene, including 2 novel variants; 3 patients had $A P O B$ mutations; and 2 patients had $A B C G 5 / G 8$ mutations. A total of $17(95 \%)$ mutation positive patients had tendon xanthomas and $13(72 \%)$ had established cardiovascular complications. In the children/adolescent group pathogenic/likely pathogenic variants were detected in 25 (89\%) patients: 21 patients had FH-causing mutations in the $L D L R$ gene, including 5 novel variants, 2 patients had $A P O B$ mutations and $A B C G 8$ and $L I P A$ mutations were found in a single patient. Additionally, $P S C K 9$ variants of unknown significance were identified in patients from both groups.

In total, 23 mutations were found in the $L D L R$ gene in both cohorts: 7 Novel variants, 3 mutations that have previously reported in Russia and 13 mutations that were identified in other populations. A total of 2 LDLR mutations were common between Saint-Petersburg and Moscow: p.(Leu401His) and
p.(Gly592Glu). These variants were detected in 3 and 6 patients, representing 5 and $10 \%$ of all FH cases reported in the present study, respectively.

Novel LDLR variants. Novel frame-shift variants in the $L D L R$ gene that lead to truncated proteins were considered certainly pathogenic. Variant c.1684_1691delTGGCCCAA p.(Pro563Hisfs*14) was found in a 16-year-old female with maximal TC levels of $9.6 \mathrm{mmol} / \mathrm{l}$. The c.433_434dupG p.(Val145Glyfs*35) variant was found in a 53-year-old woman with xanthomas and a history of myocardial infarction (MI) at the age of 53. A maximal TC level of $10.3 \mathrm{mmol} / \mathrm{l}$ was observed without statin therapy. The patient's father and uncle both died from a MI at the age of 49 and 40, respectively, and were expected to have suffered from $\mathrm{FH}$.

A novel variant in exon 8, c.1186G >C p.(Gly396Arg), was found in a young man (aged 29) who had no family history of either hyperlipidemia or ASCVD. The patient had a maximal TC level of $9.7 \mathrm{mmol} / \mathrm{l}$. During the period of genetic testing, the patient underwent a coronarography that showed preclinical diffuse atherosclerosis $(30 \%)$ of the anterior interventricular artery. The p.(Gly396Arg) variant was predicted by in silico tools as a pathogenic variant. The mutation was encoded in the EGF-like domain of LDLR, which is known to be important for receptor dissociation in endocytosis and receptor recycling to the cell surface (28), and thus could be regarded as pathogenic. It is hypothesized that this variant was functional as another similar missense variant in the codon, c.1186G $>\mathrm{A}$ p.(Gly396Ser), which had previously been found in Finland, was predicted to be disease causing (29).

Another three new missense variants encoded in the ligand-binding domain of LDLR, as well as one splicing variant, were predicted by software tools as disease causing. c.325T $>$ G p.(Cys109Gly), c.401G $>$ C p.(Cys134Ser) and c. $616 \mathrm{~A}>\mathrm{C}$, corresponding to a known protein substitution c.618T $>$ G p.(Ser206Arg), were detected in children from families with a history of hypercholesterolemia. These three patients, aged 11, 7 and 6 years old, respectively, demonstrated the highest LDL-C levels in the children/adolescent group. A splicing variant in intron 6 c.940+1_c.940+4delGTGA was detected in four unrelated individuals. This variant alters a canonical splice donor site. Previously, a similar splicing mutation, which affects 15 nucleotides (c.940_940+14del15), was described in a 21-year-old Spanish man who suffered a premature $\mathrm{MI}$ at the age of 16 years (30).

\section{Discussion}

Previously, NGS has been successfully used for mutation screening of the $L D L R$ gene and other FH-associated genes in subjects with a clinical diagnosis of definite/possible FH, as well as in patients with CAD with extremely high TC levels $(31,32)$. In the present pilot study using NGS technology for the molecular diagnosis of $\mathrm{FH}$ in Russian individuals, mutation screening of the LDLR, APOB, PSCK9, LDLRAP1, $A B C G 5 / 8, A P O E$ and $L I P A$ genes in a cohort of 59 probands was performed using NGS technology, which has been widely reported to improve the overall mutation detection rate (33-35). In the present study, pathogenic and likely pathogenic mutations were revealed in $73 \%$ of patients with definite/possible 


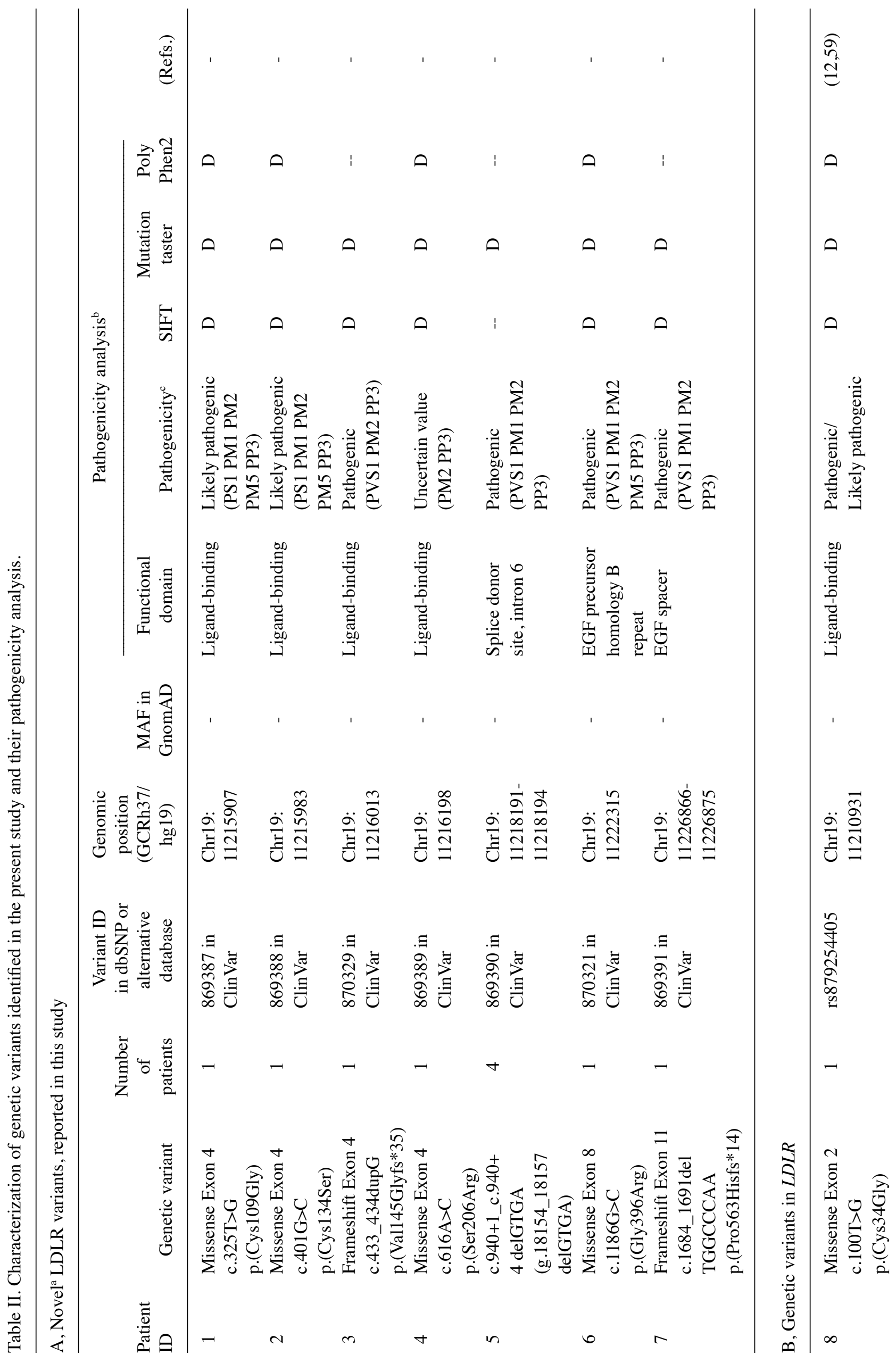




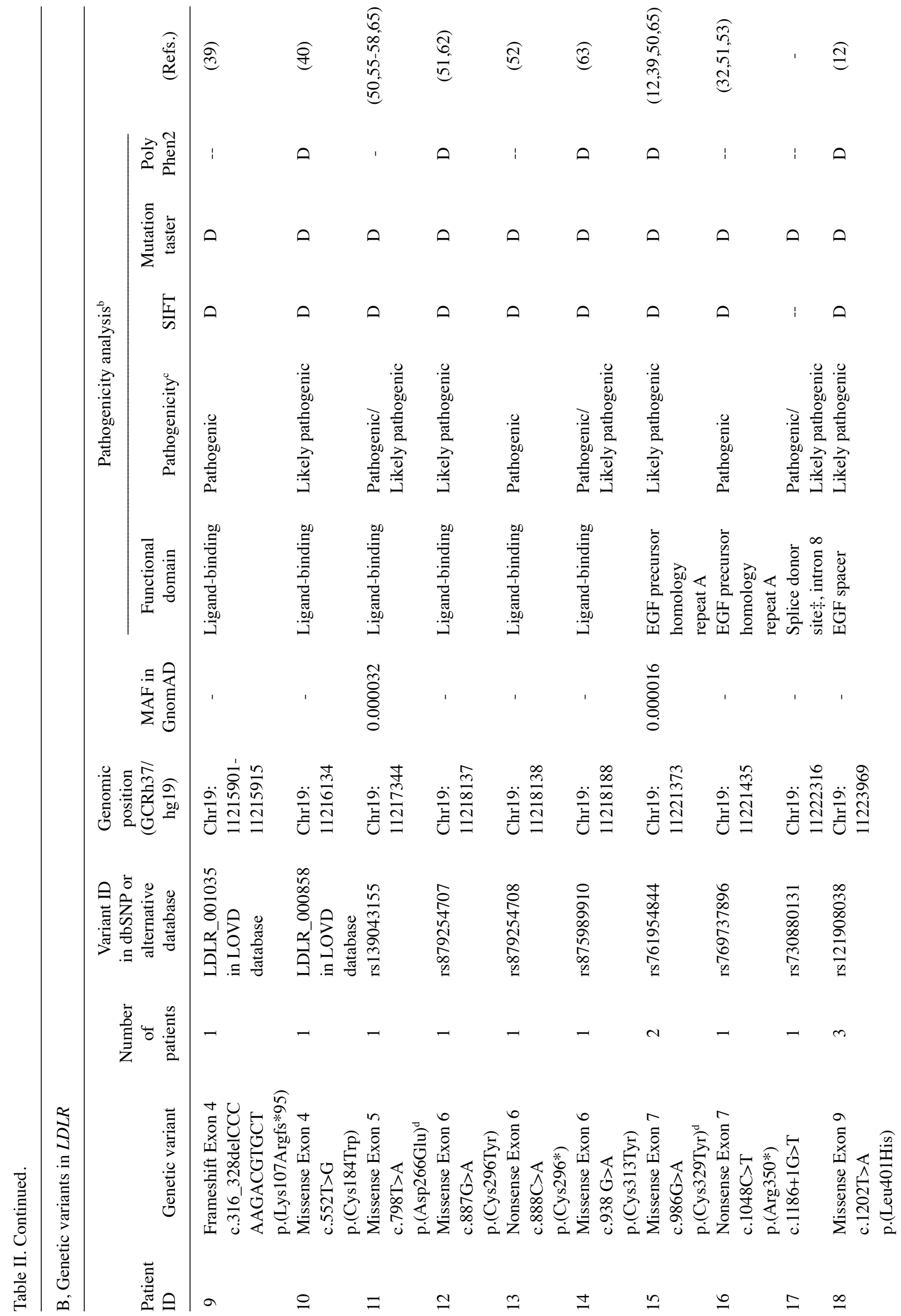




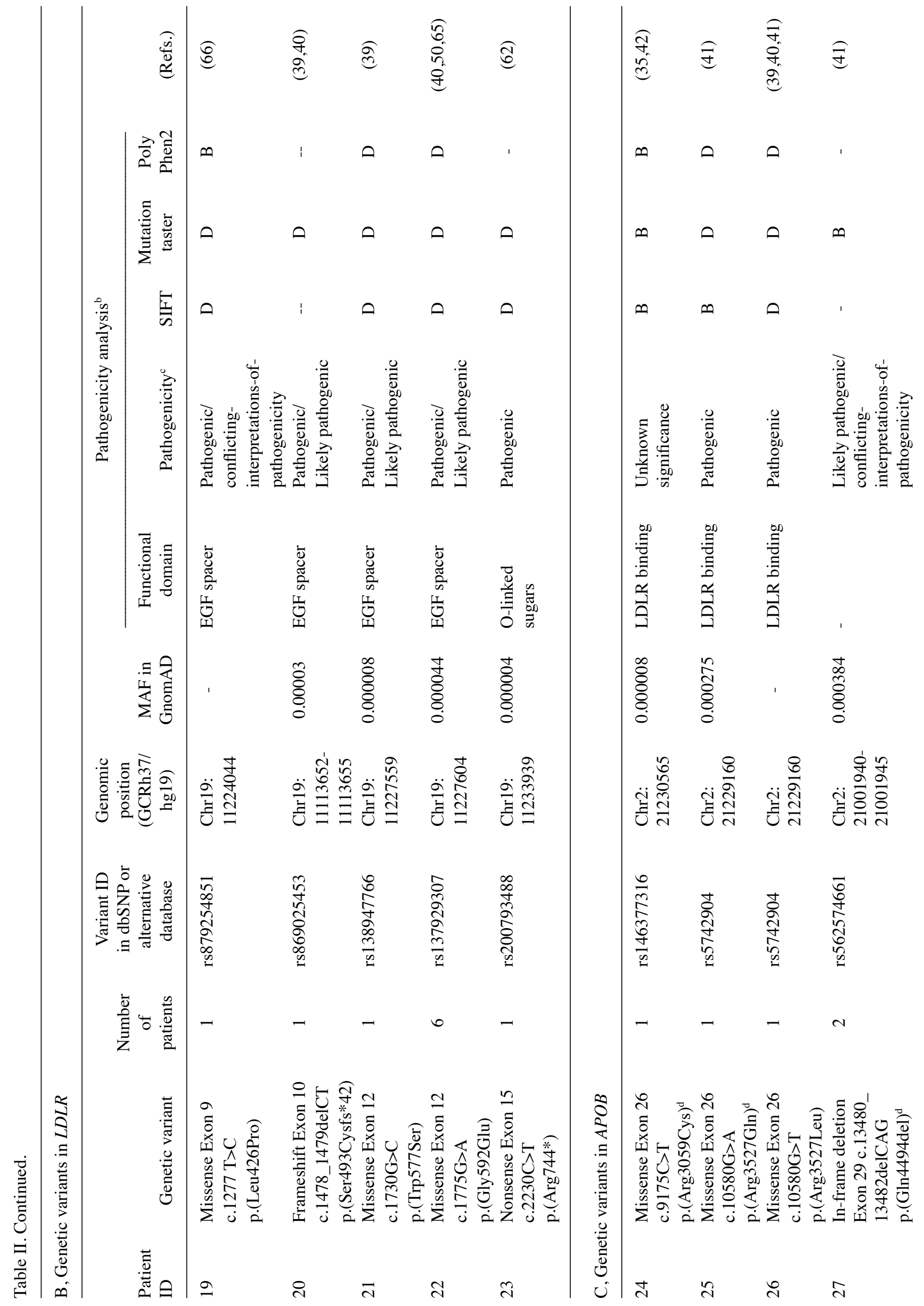




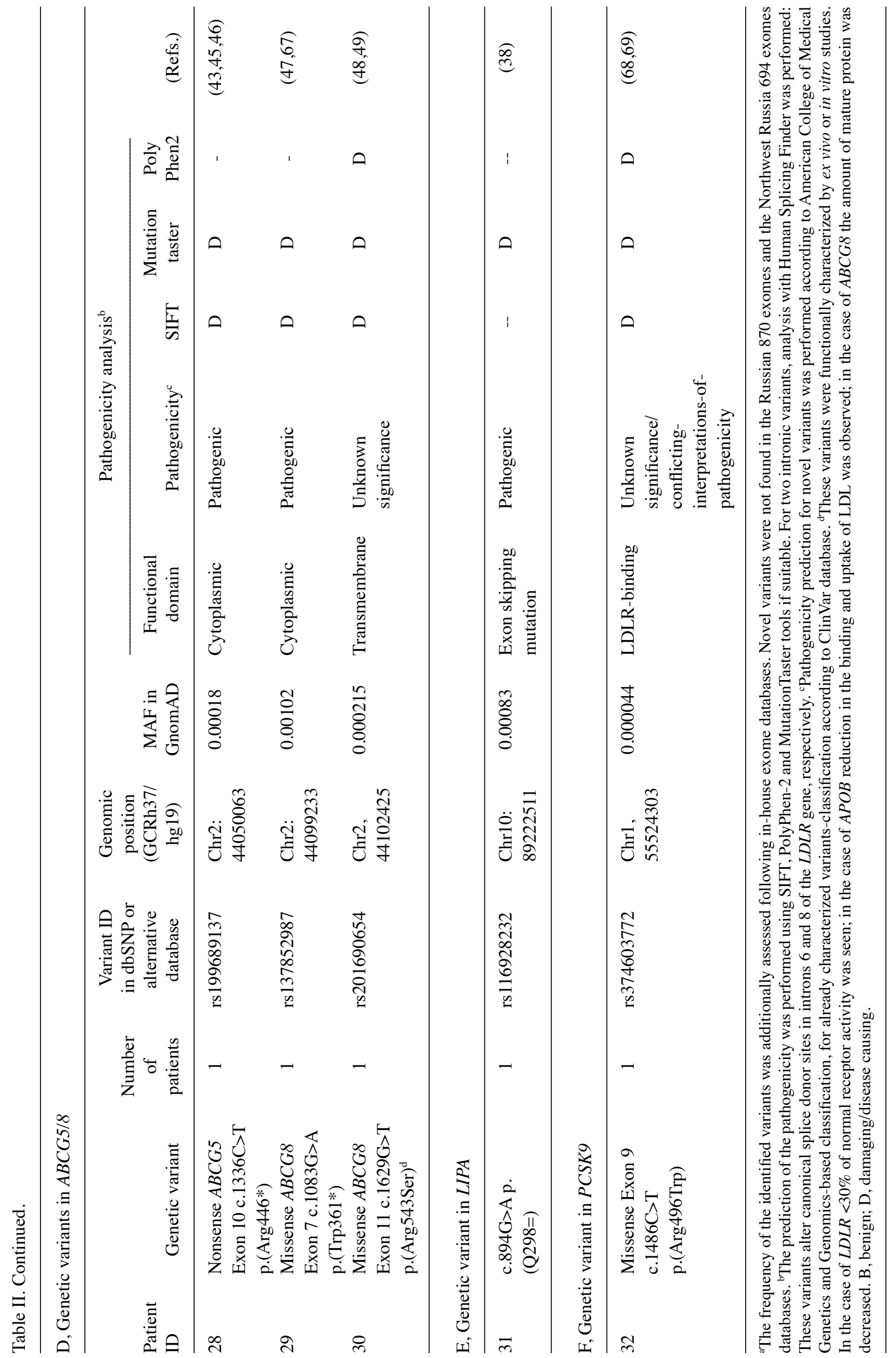


A

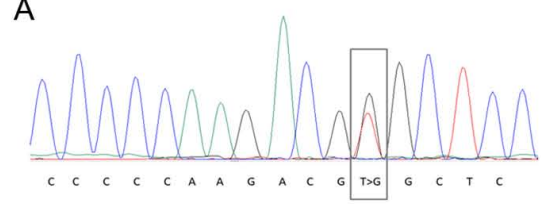

D

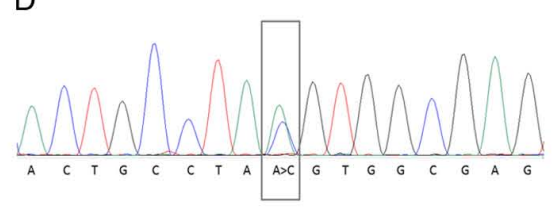

B

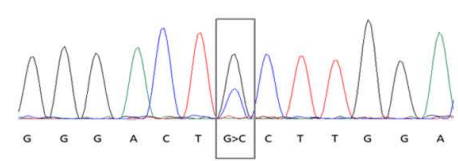

E

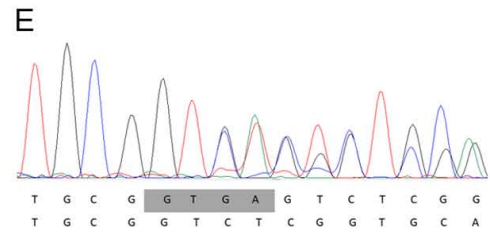

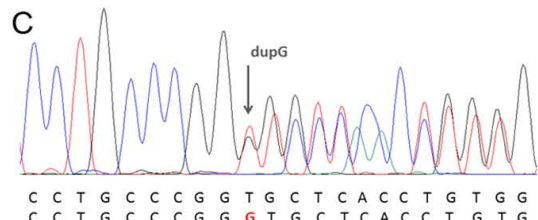

F

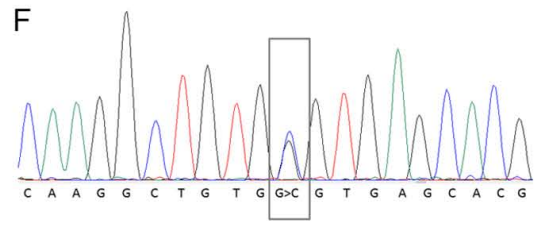

G

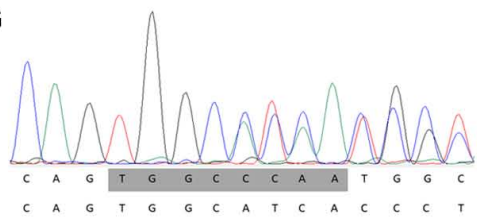

Figure 1. Sanger sequencing results for next-generation sequencing-determined novel $L D L R$ variants: (A) c.325T $>$ G p.(Cys109Gly); (B) c.401G $>$ C p.(Cys134Ser); (C) c.433_434dupG p.(Val145Glyfs*35); (D) c.616A>C p.(Ser206Arg); (E) c.940+1_c.940+4 delGTGA (g.18154_18157delGTGA); (F) c.1186G>C p.(Gly396Arg); and (G) c.1684_1691delTGGCCCAA p.(Pro563Hisfs*14).

FH. Approximately the same percentage has been commonly reported in studies where NGS technology has been applied for FH molecular diagnosis $(36,37)$. It is worth noting that previous publications conducted in Russia applying the routine methods for mutation detection with limited genes ( $L D L R$ and $A P O B$ ) have identified causative mutations in $20-50 \%$ of patients with FH $(12,14)$. Moreover, NGS technology has allowed for the inclusion of additional genes that have been linked to FH, which were previously missed by older mutation detection assays (5). The present study was, to the best of our knowledge, the first study to investigate the genetic variation in $A B C G 5 / 8$ and LIPA among Russian patients with FH.

According to a previous publication, the mutation detection rates in patients with suspected $\mathrm{FH}$ varies from $20-90 \%$, depending how rigorous the criteria used for selection of patients were (7). It should be noted that in the present study, the mutation detection rate was higher in the children/adolescent group (89\%). Only a few studies with a similar cohorts have been performed. Van der Graaf et al (7) reported that $95 \%$ of children (aged 4-18 years) with plasma LDL-C $>95$ th percentile for age and sex, and an autosomal dominant pattern for inherited hypercholesterolemia, have an FH causing mutation. This same study also reported that only $4 \%$ of children with $L D L R$ mutations exhibited physical symptoms (tendon xanthomas and an arcus cornealis), which is consistent with previous reports that have stated that only marked differences in LDL-C levels can distinguish children with FH $(6,11)$. Early childhood (1-9 years) is the optimal period for using TC or LDL-C levels to discriminate between individuals with and without FH in the general population (11). Levels of both TC and LDL-C show considerable overlap between adults with and without $\mathrm{FH}$ (17). A lower mutation detection rate in the adult group may be due to polygenic inheritance, which may explain phenotypic heterozygous FH being observed in several individuals without monogenic mutations (5).

The present study identified several novel $L D L R$ variants as well as a number of previously reported FH causing mutations in the $L D L R$ and $A P O B$ genes. These data expand upon the current knowledge of the spectrum of FH mutations in Russian individuals. Of note, it was shown that the p.(Gly592Glu) in the $L D L R$ gene may be a major $\mathrm{FH}$-associated variant for individuals from the European portion of Russia. At the same time, sequencing of noncanonical $\mathrm{FH}$ genes was established for Russian patients with $\mathrm{FH}$ and rare $A B C G 5 / 8, P S C K 9$ and LIPA mutations associated with a FH-like phenotype were found.

Identified variants were located mostly in coding regions which correspond to functional protein domains or produce truncated proteins. These variants do not overlap with any genomic regulatory regions. According to ENCODE in the UCSC Genome Browser, Tracks H3K27Ac, DNase Clusters and Txn Factor ChIP (data not shown), there was a highly probable presence of regulatory sequences in exon 1, intron 1-2, 3'-untranslated region (UTR) of the $L D L R$ gene. Introns and 3'UTR were not studied, no mutations were found in exon 1 in the present study. In the present study, 2 identified variants were predicted to alter canonical splice donor sites in introns 6 and 8 of the $L D L R$ gene. However, co-segregation analysis was not performed and there were insufficient numbers of patients to analyze linkage disequilibrium. All patients were carriers of the only one variant.

A proband with a family history of high TC levels was homozygous for a c.894G $>$ A splicing mutation in the LIPA gene, one of the most frequent variants responsible for cholesteryl ester storage disorder (CESD) (38). The variant has previously been found to have an allele frequency of $0.11 \%$ (1 in 450 individuals) in a large European population (38). This mutation is known to predominantly result in a non-functional transcript skipping of exon 8, causing the deletion of 24 amino acids (p.Q298=). CESD is associated with reduced activity of lysosomal acid lipase, an enzyme that is involved in intracellular hydrolysis of cholesteryl esters and triglycerides. It has recently been suggested that certain patients with a $\mathrm{FH}$ phenotype may have CESD (38). 
To the best of our knowledge, the present study is the first to identify mutations in the $A P O B$ gene in citizens in Saint-Petersburg. The most common mutations of the $A P O B$ gene observed in European individuals, p.(Arg3527Gln), was also found in one patient from Moscow, demonstrating an expected frequency for this region (2-4.5\%) (13). The mutation p.(Arg3527Gln) accounts for 6-10\% of all FH cases in Europe, but it was not found in the patients from Saint-Petersburg with $\mathrm{FH}$, neither in the present study nor in a previous study where screening was established for this sole mutation (12). The present study also found a rare variant in the same codon, p.(Arg3527Leu), in a patient from Saint-Petersburg. This variant has been reported in single cases from the Netherlands and Poland $(39,40)$.

As $A P O B$ mutations can be located outside the routinely analyzed $A P O B$ region, NGS allows for effective mutation screening in the entire $A P O B$ coding sequence. A rare mutation, p.(Arg3059Cys) reported in the Netherlands (35) was identified in one of the patients from Moscow, who had a family history of FH. This mutation, similar to p.(Arg3527Gln), maps to the region that binds with LDL, and the uptake of LDL particles has been shown to be significantly reduced when using LDL particles from carriers of both mutations compared with controls $(35,41)$.

A known $A P O B$ variant, p.(Gln4494del), has been detected for the first time in a Russian individual, to the best of our knowledge. This sequence results in a deletion of 3 nucleotides from exon 29 of $A P O B$ mRNA (c.13480_13482delCAG) and leads to the deletion of 1 amino acid residue of the ApoB protein, but otherwise preserves the integrity of the reading frame. The p.(Gln4494del) variant, considered as pathogenic in the UCL-FH mutation database, has been suggested as likely pathogenic or of uncertain significance in certain studies $(3,42)$. Gln4494 in the ApoB tail is important for correct protein conformation (41). Experimental studies have shown that p.(Gln4494del) causes a 40-50\% reduction in the binding and uptake of LDL. A previous study investigating secondary structure of the human ApoB using infrared spectroscopy, as well as LDL particle size using dynamic light scattering and electron microscopy, highlighted differences in the secondary structure and in the particle size of the p.(Gln4494del) variant when compared with the structure of the wild-type. These changes may underlie reduced/defective LDL binding capacity of the p.(Gln4494del) variant (41). These findings also support the notion that this mutation is disease causing.

As mutations in the $A B C G 5$ and $A B C G 8$ genes have been shown to potentially cause noticeably elevated cholesterol levels (43), these genes were included in the present analysis. One proband had an $A B C G 5$ variant p.(Arg446*) causing a premature stop codon. The patient presented with xanthomas and had a history of MI. This rare mutation which causes sitosterolemia in homozygous individuals was initially described in Italy in 2007 and later registered in Japan and Germany (44-46). High levels of blood TC and LDL-C as well as low levels of HDL-C are typical for carriers $(44,46)$. From two identified $A B C G 8$ variants, a terminating mutation p.(Trp361*) is the most common mutation causing sitosterolemia (47). The second detected variant p.(Arg543Ser) appears to cause a destabilizing substitution of conserved polar residues in the core of the transmembrane domain of
ABCG8 (48). In vitro analysis has shown that this mutation decreases the amount of mature ABCG8 protein (49). It should be noted that a patient with this mutation suffered from severe CAD and had a MI at the age of 30. Clinical manifestations of sitosterolemia can be similar to those of $\mathrm{FH}$ as $A B C G 5 / 8$ mutations are paired with extreme hypercholesterolemia (45). Notably, LDL-C levels are significantly increased in certain individuals with sitosterolemia, although the mechanism underlying this is unknown (45), and thus, those cases are occasionally misdiagnosed as FH.

In total, 16 LDLR mutations identified in the present study have been previously reported and predicted to be pathogenic/likely pathogenic. Of these, three variants have been previously found in individuals from Saint-Petersburg and Moscow, as well as in European countries: p.(Gly592Glu), p.(Cys329Tyr) and p.(Leu401His). It is notable that p.(Gly592Glu) is the most frequent mutation in Czech and Polish populations (50). The present study showed that this mutation may be frequent or may even be considered major in Russian individuals.

A further $13 L D L R$ mutations were described in Russian patients for the first time. Of these, five resulted in production of a truncated protein, and have been reported in citizens of other European countries (36,39,40,51-54). From the seven $L D L R$ missense variants identified in the present study, p.(Asp266Glu), known as FH Cincinnati-1, is the most common in Germany and Austria $(50,55)$, second most common in the Czech Republic (56) and has also been found in Denmark, Norway and USA $(57,58)$. Several loss of cysteine residues, p.(Cys34Gly), p.(Cys184Trp), p.(Cys296Tyr) and p.(Cys313Tyr), encoded in the ligand binding domain of LDLR that are associated with incorrect folding of the protein, have been previously detected in European countries $(39,40,51,58-63)$. A novel mutation from the present study, c.616A $>C$, corresponded to a known protein substitution c.618T $>$ G p.(Ser206Arg). The p.(Ser206Arg) variant is suggested to be a likely pathogenic mutation as it is located in a strongly conserved ligand-binding repeat (14). This mutation has been found in an individual from Norway and another individual from the Russian city of Petrozavodsk $(14,58)$. c.616A $>C$ leading to the same protein substitution can be assumed to be disease causing.

The present study has some limitations. For example, analysis of introns/3'UTRs of the studied genes was not performed, and co-segregation analysis could not be performed due to an insufficient number of patients for linkage disequilibrium studies.

In conclusion, the mutation spectrum for $\mathrm{FH}$ in Russian individuals is similar to that of other European countries. Evidence of this conclusion is that certain $L D L R$ mutations that had initially been found in Russian individuals $(12,64)$ were subsequently identified in European populations, such as p.(Cys249*), p.(Trp422*), p.(Asp601Asn) and p.(Arg410Gly) $(39,40,50,51)$. Mutations in the $L D L R$ gene are very diverse and can be present in any part of the gene; therefore, it was not unexpected that through using NGS, novel $L D L R$ variants were discovered in the present study. NGS allowed for the investigation of an extended list of FH associated genes, and for the first time revealed rare mutations in the $A B C G 5 / 8$ and LIPA genes in Russian patients with FH. 


\section{Acknowledgements}

Not applicable.

\section{Funding}

This work was partly supported by the Russian Science Foundation (grant no. 14-50-00069) and the state assignment of Ministry of science and Higher Education of the Russian Federation for RCMG.

\section{Availability of data and materials}

The data that support the findings of this study are available from the corresponding author upon reasonable request. The data are not publicly available due to privacy or ethical restrictions. All novel variants have been submitted to the ClinVar database (ncbi.nlm.nih.gov/clinvar/; IDs: 869387, 869388, 869389, 869390, 869391, 870321, 870329).

\section{Author's contributions}

EYZ, OSG, ASG, AMS, SNP designed and conceived the methodology of the present study, organized experiments and performed final interpretation of the data. MVM, SAU, VSG, SPU, SGS, IVA and DMG collected the patient data and assisted with clinical data interpretation. OVR, ONI, NAS, MAF and VVM performed the experiments. YAB, AAP, VVM, MAF and ONI performed the bioinformatics analysis and variant annotation. VVM wrote the first draft of the manuscript. All authors read and approved the final manuscript.

\section{Ethics approval and consent to participate}

This study was approved by the Ethics Committees of Center for Atherosclerosis and Lipid Disorders of North-Western District Scientific and Clinical Center Named After L.G. Sokolov, Medical Faculty of Saint-Petersburg State University, City Hospital No. 40 (St. Petersburg, Russia) and Research Centre for Medical Genetics (Moscow, Russia) where patients were treated and genetic analysis was performed. Written informed consent was obtained from all patients or their legal representatives before the study.

\section{Patient consent for publication}

Not applicable.

\section{Competing interests}

The authors declare that they have no competing interests.

\section{References}

1. Defesche JC, Gidding SS, Harada-Shiba M, Hegele RA, Santos RD and Wierzbicki AS: Familial hypercholesterolaemia. Nat Rev Dis Primers 3: 17093, 2017.

2. Di Taranto MD, Giacobbe $\mathrm{C}$ and Fortunato G: Familial hypercholesterolemia: A complex genetic disease with variable phenotypes. Eur J Med Genet 63: 103831, 2020.
3. Sharifi M, Futema M, Nair D and Humphries SE: Genetic architecture of familial hypercholesterolaemia. Curr Cardiol Rep 19: 44, 2017.

4. Berge KE, Tian H, Graf GA, Yu L, Grishin NV, Schultz J, Kwiterovich P, Shan B, Barnes R and Hobbs HH: Accumulation of dietary cholesterol in sitosterolemia caused by mutations in adjacent ABC transporters. Science 290: 1771-1775, 2000.

5. Iacocca MA and Hegele RA: Recent advances in genetic testing for familial hypercholesterolemia. Expert Rev Mol Diagn 17: 641-651, 2017.

6. Wiegman A, Gidding SS, Watts GF, Chapman MJ, Ginsberg HN, Cuchel M, Ose L, Averna M, Boileau C, Borén J, et al: European atherosclerosis society consensus panel. Familial hypercholesterolaemia in children and adolescents: Gaining decades of life by optimizing detection and treatment. Eur Heart J 36: 2425-2437, 2015.

7. Van der Graaf A, Avis HJ, Kusters DM, Vissers MN, Hutten BA, Defesche JC, Huijgen R, Fouchier SW, Wijburg FA, Kastelein JJ and Wiegman A: Molecular basis of autosomal dominant hypercholesterolemia: Assessment in a large cohort of hypercholesterolemic children. Circulation 123: 1167-1173, 2011.

8. Santos RD, Gidding SS, Hegele RA, Cuchel MA, Barter PJ, Watts GF, Baum SJ, Catapano AL, Chapman MJ, Defesche JC, et al: Defining severe familial hypercholesterolaemia and the implications for clinical management: A consensus statement from the international atherosclerosis society severe familial hypercholesterolemia panel. Lancet Diabetes Endocrinol 4: 850-861, 2016.

9. Harada-Shiba M, Ohta T, Ohtake A, Ogura M, Dobashi K, Nohara A, Yamashita S and Yokote K; Joint Working Group by Japan Pediatric Society and Japan Atherosclerosis Society for Making Guidance of Pediatric Familial Hypercholesterolemia: Guidance for pediatric familial hypercholesterolemia 2017. J Atheroscler Thromb 25: 539-553, 2018.

10. Ramaswamia U, Humphries SE, Priestley-Barnhamc L, Green P, Wald DS, Capps N, Andersong M, Dale P and Morris AA: Current management of children and young people with heterozygous familial hypercholesterolaemia-HEART UK statement of care. Atherosclerosis 290: 1-8, 2019.

11. Martin AC, Gidding SS, Wiegman A and Watts GF: Knowns and unknowns in the care of pediatric familial hypercholesterolemia. J Lipid Res 58: 1765-1776, 2017.

12. Zakharova FM, Damgaard D, Mandelshtam MY, Golubkov VI, Nissen PH, Nilsen GG, Stenderup A, Lipovetsky BM, Konstantinov VO, Denisenko AD, et al: Familial hypercholesterolemia in St-Petersburg: The known and novel mutations found in the low density lipoprotein receptor gene in Russia. BMC Med Genet 6: 6, 2005 .

13. Malyshev PP, Meshkov AN, Kotova LA and Kuharchuk VV: Familial defect of apolipoprotein B-100: Molecular disease basis and clinic-biochemical characteristics of the patients. Cardiovascular Ther Prevention 6: 40-45, 2007 (In Russian).

14. Korneva VA, Kuznetsova TY, Golovina AS, Vasilyev VB and Mandelshtam MY: Familial hypercholesterolemia mutations in Petrozavodsk: No similarity to St. Petersburg mutation spectrum. BMC Med Genet 14: 128, 2013.

15. Hardin AP and Hackell JM; Committee on Practice and Ambulatory Medicine: Age limits in pediatrics. Pediatrics 140: e20172151, 2017.

16. Watts GF, Sullivan DR, Poplawski N, van Bockxmeer F, Hamilton-Craig I, Clifton PM, O'Brien R, Bishop W, George P, Barter PJ, et al: Familial hypercholesterolaemia: A model of care for Australasia. Atheroscler Suppl 12: 221-263, 2011.

17. Di Taranto MD, de Falco R, Guardamagna O, Massini G, Giacobbe C, Auricchio R, Malamisura B, Proto M, Palma D, Greco L and Fortunato G: Lipid profile and genetic status in a familial hypercholesterolemia pediatric population: Exploring the LDL/HDL ratio. Clin Chem Lab Med 57: 1102-1110, 2019.

18. 1000 Genomes Project Consortium; Auton A, Brooks LD, Durbin RM, Garrison EP, Kang HM, Korbel JO, Marchini JL, McCarthy S, McVean GA and Abecasis GR: A global reference for human genetic variation. Nature 526: 68-74, 2015.

19. DePristo MA, Banks E, Poplin R, Garimella KV, Maguire JR, Hartl C, Philippakis AA, del Angel G, Rivas MA, Hanna M, et al: A framework for variation discovery and genotyping using next-generation DNA sequencing data. Nat Genet 43: 491-498, 2011.

20. Van der Auwera GA, Carneiro MO, Hartl C, Poplin R, Del Angel G, Levy-Moonshine A, Jordan T, Shakir K, Roazen D, Thibault J, et al: From Fastq data to high-confidence variant calls: The genome analysis toolkit best practices pipeline. Curr Protoc Bioinformatics 43: 11.10.1-11.10.33, 2013. 
21. Auton A, Brooks LD, Durbin RM, Garrison EP, Kang HM Korbel JO, Marchini JL, McCarthy S, McVean GA and Abecasis GR: A global reference for human genetic variation. Nature 526: 68-74, 2015.

22. Lek M, Karczewski KJ, Minikel EV and Samocha KE: Analysis of protein-coding genetic variation in 60,706 humans. Nature 536: 285-291, 2016.

23. Liu X, Wu C, Li C, Boerwinkle E, Jolla L and Genome H: dbNSFP v3.0: A one-stop database of functional predictions and annotations for human non-synonymous and splice site SNVs. Hum Mutat 37: 235-241, 2016

24. Barbitoff YA, Skitchenko RK, Poleshchuk OI, Shikov AE, Serebryakova EA, Nasykhova YA, Polev DE, Shuvalova AR, Shcherbakova IV, Fedyakov MA, et al: Whole-exome sequencing provides insights into monogenic disease prevalence in Northwest Russia. Mol Genet Genomic Med 7: e964, 2019.

25. Desmet FO, Hamroun D, Lalande M, Collod-Béroud G, Claustres $\mathrm{M}$ and Béroud $\mathrm{C}$ : Human splicing finder: An online bioinformatics tool to predict splicing signals. Nucleic Acids Res 37: e67, 2009.

26. Richards S, Aziz N, Bale S, Bick D, Das S, Gastier-Foster J, Grody WW, Hegde M, Lyon E, Spector E, et al: Standards and guidelines for the interpretation of sequence variants: $A$ joint consensus recommendation of the American College of Medical Genetics and Genomics and the Association for Molecular Pathology. Genet Med 17: 405-424, 2015.

27. Glotov OS, Serebryakova EA, Turkunova MS, Efimova OA, Glotov AS, Barbitoff YA, Nasykhova YA, Predeus AV, Polev DE, Fedyakov MA, et al: Whole-exome sequencing for monogenic diabetes in Russian children reveals wide spectrum of genetic variants in MODY-related and unrelated genes. Mol Med Rep 20: 4905-4914, 2019

28. Arias-Moreno X, Velazquez-Campoy A, Rodríguez JC, Pocoví M and Sancho J: Mechanism of low density lipoprotein (LDL) release in the endosome: Implications of the stability and $\mathrm{Ca} 2+$ affinity of the fifth binding module of the LDL receptor. J Biol Chem 283: 22670-22679, 2008.

29. Koivisto UM, Viikari JS and Kontula K: Molecular characterization of minor gene rearrangements in Finnish patients with heterozygous familial hypercholesterolemia: Identification of two common missense mutations (Gly823->Asp and Leu380->His) and eight rare mutations of the LDL receptor gene. Am J Hum Genet 57: 789-797, 1995.

30. Maglio C, Mancina RM, Motta BM, Stef M, Pirazzi C, Palacios L, Askaryar N, Boren J, Wiklund O and Romeo S: Genetic diagnosis of familial hypercholesterolaemia by targeted next-generation sequencing. J Intern Med 276: 396-403, 2014.

31. Norsworthy PJ, Vandrovcova J, Thomas ERA, Campbell A, Kerr SM, Biggs J, Game L, Soutar AK, Smith BH, Dominiczak AF, et al: Targeted genetic testing for familial hypercholesterolaemia using next generation sequencing: A population-based study. BMC Med Genet 15: 70, 2014.

32. Radovica-Spalvina I, Latkovskis G, Silamikelis I, Fridmanis D, Elbere I, Ventins KV, Ozola G, Erglis A and Klovins J: Next-generation-sequencing-based identification of familial hypercholesterolemiarelated mutations in subjects with increased LDL-C levels in a latvian population. BMC Med Genet 16: 86, 2015.

33. Johansen CT, Dubé JB, Loyzer MN, MacDonald A, Carter DE, McIntyre AD, Cao H, Wang J, Robinson JF and Hegele RA: LipidSeq: A next-generation clinical resequencing panel for monogenic dyslipidemias. J Lipid Res 55: 765-772, 2014.

34. Vandrovcova J, Thomas ER, Atanur SS, Norsworthy PJ, Neuwirth C, Tan Y, Kasperviciute D, Biggs J, Game L, Mueller M, et al: The use of next-generation sequencing in clinical diagnosis of familial hypercholesterolemia. Genet Med 15: 948-957, 2013.

35. Motazacker MM,Pirruccello J,Huijgen R, Do R, Gabriel S,Peter J, Kuivenhoven JA, Defesche JC, Kastelein JJ, Hovingh GK, et al Advances in genetics show the need for extending screening strategies for autosomal dominant hypercholesterolaemia. Eur Heart J 33: 1360-1366, 2012

36. Reiman A, Pandey S, Lloyd KL, Dyer N, Khan M, Crockard M, Latten MJ, Watson TL, Cree IA and Grammatopoulos DK Molecular testing for familial hypercholesterolaemia-associated mutations in a UK-based cohort: Development of an NGS-based method and comparison with multiplex polymerase chain reaction and oligonucleotide arrays. Ann Clin Biochem 53: 654-662, 2016.

37. Wang J, Dron JS, Ban MR, Robinson JF, Mc Intyre AD, Alazzam M, Zhao PJ, Dilliott AA, Cao H, Huff MW, et al: Polygenic versus monogenic causes of hypercholesterolemia ascertained clinically. Arterioscler Thromb Vasc Biol 36: 2439-2445, 2016
38. Ashfield-Watt P, Haralambos K, Edwards R, Townsend D, Gingell R, Wa Li K, Humphries SE and McDowell I: Estimation of the prevalence of cholesteryl ester storage disorder in a cohort of patients with clinical features of familial hypercholesterolaemia. Ann Clin Biochem 56: 112-117, 2019.

39. Fouchier SW, Kastelein JJ and Defesche JC: Update of the molecular basis of familial hypercholesterolemia in The Netherlands. Hum Mutat 26: 550-556, 2005.

40. Chmara M, Wasag B, Zuk M, Kubalska J, Wegrzyn A, Bednarska-Makaruk M, Pronicka E, Wehr H, Defesche JC, Rynkiewicz A and Limon J: Molecular characterization of Polish patients with familial hypercholesterolemia: Novel and recurrent LDLR mutations. J Appl Genet 51: 95-106, 2010.

41. Fernández-Higuero JA, Etxebarria A, Benito-Vicente A, Alves AC, Arrondo JL, Ostolaza H, Bourbon M and Martin C: Structural analysis of APOB variants, p.(Arg3527Gln), p.(Arg1164Thr) and p.(Gln4494del), causing Familial Hypercholesterolaemia provides novel insights into variant pathogenicity. Sci Rep 5: 18184, 2015.

42. Alves AC, Etxebarria A, Soutar AK, Martin C and Bourbon M: Novel functional APOB mutations outside LDL-binding region causing familial hypercholesterolaemia. Hum Mol Genet 23: 1817-1828, 2013

43. Rios J, Stein E, Shendure J, Hobbs HH and Cohen JC: Identification by whole-genome resequencing of gene defect responsible for severe hypercholesterolemia. Hum Mol Genet 19: 4313-4318, 2010.

44. Mannucci L, Guardamagna O, Bertucci P, Pisciotta L, Liberatoscioli L, Bertolini S, Irace C, Gnasso A, Federici G and Cortese C: Beta-sitosterolaemia: A new nonsense mutation in the ABCG5 gene. Eur J Clin Invest 37: 997-1000, 2007.

45. Tada H, Kawashiri MA, Takata M, Matsunami K, Imamura A, Matsuyama M, Sawada H, Nunoi H, Konno T, Hayashi K, Nohara A, et al: Infantile cases of Sitosterolaemia with novel mutations in the ABCG5 gene: Extreme hypercholesterolaemia is exacerbated by breastfeeding. JIMD Rep 21: 115-122, 2015.

46. Li Y, Salfelder A, Schwab KO, Grünert SC, Velten T, Lütjohann D, Villavicencio-Lorini P, Matysiak-Scholze U, Zabel B, Köttgen A and Lausch E: Against all odds: Blended phenotypes of three single-gene defects. Eur J Hum Genet 24: 1274-1279, 2016.

47. Lu K, LeeMH, Hazard S, Brooks-Wilson A, Hidaka H, Kojima H, Ose L, Stalenhoef AF, Mietinnen T, Bjorkhem I, et al: Two Genes that map to the STSL locus cause Sitosterolemia: Genomic structure and spectrum of mutations involving Sterolin-1 and Sterolin-2, encoded by ABCG5 and ABCG8, respectively. Am J Hum Genet 69: 278-290, 2001.

48. Lee JY, Kinch LN, Borek DM, Wang J, Wang J, Urbatsch IL, Xie XS, Grishin NV, Cohen JC, Otwinowski Z, et al: Crystal structure of the human sterol transporter ABCG5/ABCG8. Nature 533: 561-564, 2016.

49. Graf GA, Cohen JC and Hobbs HH: Missense mutations in ABCG5 and ABCG8 disrupt heterodimerization and trafficking. J Biol Chem 279: 24881-24888, 2004.

50. Tichý L, Freiberger T, Zapletalová P, Soska V, Ravcuková B and Fajkusová L: The molecular basis of familial hypercholesterolemia in the Czech Republic: Spectrum of LDLR mutations and genotype-phenotype correlations. Atherosclerosis 223: 401-408, 2012.

51. Bertolini S, Pisciotta L, Rabacchi C, Cefalù AB, Noto D, Fasano T, Signori A, Fresa R, Averna $M$ and Calandra S: Spectrum of mutations and phenotypic expression in patients with autosomal dominant hypercholesterolemia identified in Italy. Atherosclerosis 227: 342-348, 2013.

52. Lind S, Eriksson M, Rystedt E, Wiklund O, Angelin B and Eggertsen G: Low frequency of the common Norwegian and Finnish LDL-receptor mutations in Swedish patients with familial hypercholesterolaemia. J Intern Med 244: 19-25, 1998.

53. Solberg K, Rødningen OK, Tonstad S, Ose L and Leren TP: Familial hypercholesterolaemia caused by a non-sense mutation in codon 329 of the LDL receptor gene. Scand J Clin Lab Invest 54: 605-609, 1994.

54. Górski B, Kubalska J, Naruszewicz M and Lubiński J: LDL-R and Apo-B-100 gene mutations in Polish familial hypercholesterolemias. Hum Genet 102: 562-565, 1998.

55. Brænne I, Kleinecke M, Reiz B, Graf E, Strom T, Wieland T, Fischer M, Kessler T, Hengstenberg C, Meitinger T, et al: Systematic analysis of variants related to familial hypercholesterolemia in families with premature myocardial infarction. Eur J Hum Genet 24: 191-197, 2016. 
56. Kuhrová V, Francová H, Zapletalová P, Freiberger T, Fajkusová L, Hrabincová E, Slováková R and Kozák L: Spectrum of low density lipoprotein receptor mutations in Czech hypercholesterolemic patients. Hum Mutat 18: 253, 2001.

57. Brusgaard K, Jordan P, Hansen H, Hansen AB and Hørder M Molecular genetic analysis of 1053 Danish individuals with clinical signs of familial hypercholesterolemia. Clin Genet 69 277-283, 2006

58. Leren TP, Manshaus T, Skovholt U, Skodje T, Nossen IE, Teie C, Sørensen S and Bakken KS: Application of molecular genetics for diagnosing familial hypercholesterolemia in Norway: Results from a family-based screening program. Semin Vasc Med 4: $75-85,2004$.

59. Duskova L, Kopeckova L, Jansova E, Tichy L, Freiberger T, Zapletalova P, Soskac V, Ravcukova B and Fajkusova L: An APEX-based genotyping microarray for the screening of 168 mutations associated with familial hypercholesterolemia. Atherosclerosis 216: 139-145, 2011.

60. Lind S, Rystedt E, Eriksson M, Wiklund O, Angelin B and Eggertsen G: Genetic characterization of Swedish patients with familial hypercholesterolemia: A heterogeneous pattern of mutations in the LDL receptor gene. Atherosclerosis 163: 399-407, 2002.

61. Nauck MS, Köster W, Dörfer K, Eckes J, Scharnagl H, Gierens H, Nissen H, Nauck MA, Wieland H and März W: Identification of recurrent and novel mutations in the LDL receptor gene in German patients with familial hypercholesterolemia. Hum Mutat 18: 165-166, 2001.

62. Marduel M, Carrié A, Sassolas A, Devillers M, Carreau V, Di Filippo M, Erlich D, Abifadel M, Marques-Pinheiro A, Munnich A, et al: Molecular spectrum of autosomal dominant hypercholesterolemia in France. Hum Mutat 31: E1811-E1824, 2010.

63. Day IN, Whittall RA, O'Dell SD, Haddad L, Bolla MK, Gudnason V and Humphries SE: Spectrum of LDL receptor gene mutations in heterozygous familial hypercholesterolemia. Hum Mutat 10: 116-127, 1997.
64. Voevoda MI, Kulikov IV, Shakhtshneider EV, Maksimov VN, Pilipenko IV, Tereschenkov IP, Kobzev VF, Romaschenko AG and Nikitin YP: The spectrum of mutations in the low-density lipoprotein receptor Gene in the Russian Population. Genetics 44: 1191-1194, 2008

65. Benito-Vicente A, Uribe KB, Jebari S, Galicia-Garcia U, Ostolaza $\mathrm{H}$ and Martin C: Validation of LDLr Activity as a tool to improve genetic diagnosis of familial hypercholesterolemia: A retrospective on functional characterization of LDLr variants. Int J Mol Sci 19: 1676, 2018.

66. Mak YT, Pang CP, Tomlinson B, Zhang J, Chan YS, Mak TW and Masarei JR: Mutations in the low-density lipoprotein receptor gene in Chinese familial hypercholesterolemia patients. Arterioscler Thromb Vasc Biol 18: 1600-1605, 1998.

67. Hansel B, Carrié A, Brun-Druc N, Leclert G, Chantepie S, Coiffard AS, Kahn JF, Chapman MJ and Bruckert E: Premature atherosclerosis is not systematic in phytosterolemic patients: Severe hypercholesterolemia as a confounding factor in five subjects. Atherosclerosis 234: 162-168, 2014.

68. Du F, Hui Y, Zhang M, Linton MF, Fazio S and Fan D Novel Domain Interaction Regulates Secretion of Proprotein Convertase Subtilisin/Kexin Type 9 (PCSK9) Protein. J Biol Chem 286: 43054-43061, 2011.

69. Kaya E, Kayikçioğlu M, Vardarli AT, Eroğlu Z, Payzın S and Can L: PCSK 9 gain-of-function mutations (R496W and D374Y) and clinical cardiovascular characteristics in a cohort of Turkish patients with familial hypercholesterolemia. Anatol J Cardiol 18: 266-272, 2017.

This work is licensed under a Creative Commons Attribution-NonCommercial-NoDerivatives 4.0 International (CC BY-NC-ND 4.0) License. 